\title{
Controlling for dilution while manipulating load: Perceptual and sensory limitations are just two aspects of task difficulty
}

\author{
Hanna Benoni • Yehoshua Tsal
}

Published online: 31 March 2012

(C) Psychonomic Society, Inc. 2012

\begin{abstract}
Lavie and de Fockert (2003) proposed that perceptual load and sensory limitations reflect two distinct mechanisms producing opposite effects: Perceptual load eliminates distractor interference, whereas sensory limitations enhance it. Tsal and Benoni (2010a) suggested that these results may have been due to the confounding effect of dilution - that is, to the presence (perceptual load) or absence (sensory load) of neutral items capable of diluting distractor interference. In the present study, we jointly manipulated dilution with perceptual load and with sensory degradation. The results show that, with both perceptual and sensory manipulations, the presence of diluting items eliminates distractor interference, whereas controlling for dilution increases distractor interference. We therefore conclude that perceptual and sensory limitations are just two aspects of task difficulty.
\end{abstract}

Keywords Selective attention · Spatial attention

The theory of perceptual load (Lavie, 1995; Lavie \& Tsal, 1994) proposes that the level of perceptual load of relevant processing determines the level of interference of irrelevant distractors. Thus, irrelevant information will be excluded from processing only if the prioritized relevant processing exhausts all of the available resources. When the relevant stimuli do not demand all of the available attentional capacity, irrelevant stimuli will unintentionally capture spare capacity, consequently enabling their processing.

H. Benoni $(\bowtie) \cdot$ Y. Tsal

Department of Psychology, Tel Aviv University,

Ramat Aviv, Tel Aviv 69978, Israel

e-mail: hannib@bezeqint.net
Over the past decade, perceptual-load theory has received a great deal of attention and has had a tremendous impact on visual attention (e.g., Bavelier, Deruelle, \& Proksch, 2000; Beck \& Lavie, 2005; Kumada \& Humphreys, 2002; Lavie, 1995; Lavie \& Cox, 1997; Lavie \& Fox, 2000; Maylor \& Lavie, 1998; Rees, Frith, \& Lavie, 1997, 2001). Moreover, this impact has extended beyond the attention literature, as it has been claimed that perceptual load is a dominating mechanism mediating a variety of perceptual and cognitive operations, such as negative priming (Lavie \& Fox, 2000), change blindness (Lavie, 2006), interference of taskunrelated stimuli (Forster \& Lavie, 2008), modification of the startle reflex (Thorne, Dawson, \& Schell, 2006), flicker detection (Carmel, Saker, Rees, \& Lavie, 2007), and a host of other perceptual and cognitive phenomena.

In typical manipulations of perceptual load, the target and distractor appear alone in the low-load condition but in the presence of additional, neutral elements in the high-load condition. It has therefore been argued recently (Benoni \& Tsal, 2010; Tsal \& Benoni, 2010a, 2010b; Wilson, Muroi, \& MacLeod, 2011) that the reduction of distractor interference under high-load conditions need not be attributed to increases in perceptual load that result from the need to search for the target among the neutral letters. Instead, the reduction could be due to the dilution of the distractor by the neutral letters, as the representations of their features are highly activated in the process of searching for the target. Indeed, three different studies (Benoni \& Tsal, 2010; Tsal \& Benoni, 2010a; Wilson et al., 2011) distinguished between the possible effects of perceptual load and dilution by introducing low-load, high-dilution displays. These displays contained neutral letters (as in high-load conditions) capable of diluting the distractor. Yet either the stimulus or the processing requirements allowed for a low-load processing mode. For example, in a multiple-color display, the 
target color was preknown in the low-load, high-dilution condition, but not in the high-load condition (Tsal \& Benoni, 2010a). In all experiments, using a variety of converging operations, distractor processing was completely eliminated for these new displays, thereby supporting the conclusion that the elimination of distractor interference under high-load conditions, traditionally attributed to perceptual load, is completely accounted for by dilution. The alternative, dilution interpretation has received further support from subsequent studies (e.g., Dittrich \& Stahl, 2011; Kyllingsbaek, Sy, \& Giesbrecht, 2011; Marciano \& Yeshurun, 2011).

The purpose of the present study was to investigate the possibility that dilution can also explain an important extension of perceptual-load theory - namely, the opposite effects obtained via manipulations of perceptual load and sensory degradation. Lavie and de Fockert (2003) attempted to further substantiate load theory by distinguishing perceptual load from general task difficulty. Specifically, they contrasted the effects of perceptual load (increasing display size) with those of sensory degradation (reducing the size, contrast, duration, and retinal acuity of a target). The major finding of this study was that, while perceptual load reduced distractor interference, sensory load substantially increased it. It is important to note that while the difference between sensory load and perceptual load is not at all clear (as will be detailed in the General Discussion below), one clear difference is apparent between the perceptual and sensory manipulations used by Lavie and de Fockert: To increase perceptual load, additional neutral (potentially diluting) letters were added to the display, whereas to increase sensory load, the target was presented alone, without the accompanying neutral letters. Hence, one straightforward interpretation of these results would be in terms of a dilution effect. That is, distractor interference is diminished only when task difficulty is increased by adding neutral letters that can dilute that interference (perceptual load), and not when the task difficulty is increased but the target remains by itself (sensory degradation). The dilution interpretation leads to two predictions tested in the present study. First, if potentially diluting items are added to the display with the degraded target, distractor interference ought to be substantially reduced (Exp. 1). Second, if the effect of dilution is controlled, increasing perceptual load should increase distractor interference similar to increasing sensory degradation (Exp. 2).

\section{Experiment 1}

In Experiment 1, we tested the prediction that the increased distractor interference resulting from degrading the target will be largely reduced in the presence of additional, neutral letters capable of diluting distractor processing. This experiment included four conditions: low perceptual load, high perceptual load, sensory degradation, and sensory degradation with dilution. The first three conditions above are the ones tested in Lavie and de Fockert's (2003) study. The fourth condition was added in order to test the alternative, dilution interpretation. In addition to the degraded target, it included additional neutral letters similar to the nontarget letters present in the high-perceptual-load condition. In all conditions, the target was presented in one of six possible circular positions. A larger distractor was presented in the left or right periphery. The five nontarget circular positions either remained empty (in the conditions of low perceptual load and sensory degradation) or were occupied by five neutral letters (in the conditions of high perceptual load and sensory degradation with dilution). In the two sensorydegradation conditions, the target letter was substantially reduced in size and in contrast, in a manner similar to the sensory manipulation of Lavie and de Fockert (2003).

\section{Method}

\section{Participants}

The participants were 12 undergraduates from Tel Aviv University, who participated to fulfill a course requirement. All had normal or corrected-to-normal vision.

\section{Stimuli and procedure}

The experiment was conducted in a dimly lit room. Displays were generated by an IBM PC computer attached to a 17-in. monitor, and responses were collected via the computer keyboard. A chinrest was used to stabilize the viewing distance at $60 \mathrm{~cm}$ from the monitor. The stimuli were presented on a black background and were all light gray (color $16 \mathrm{X} 8$ in the palette), except for the target letters in the two sensory-degradation conditions, which were dark gray (color 16X14 in the palette). Examples of the stimulus displays are presented in Fig. 1. Each display consisted of a target letter, either an $\mathrm{X}$ or an $\mathrm{N}$, subtending $0.72 \mathrm{deg}$ in height and $0.54 \mathrm{deg}$ in width in the low-load and high-load conditions, or $0.37 \mathrm{deg}$ in height and $0.31 \mathrm{deg}$ in width in the two sensory-degradation conditions. An irrelevant distractor subtending $0.96 \mathrm{deg}$ in height and $0.64 \mathrm{deg}$ in width was also presented in each display. The distractor was also either an X or an N. Thus, the distractor could either be congruent (identical to the target) or incongruent (identical to the nonpresented target). The two possible target letters appeared equally frequently and were randomly intermixed. For each target letter, the two possible distractors were also equally frequent and randomly intermixed. The target letter was randomly presented in one of six possible locations, arranged on an imaginary circle subtending $2.48 \mathrm{deg}$ of visual 
Fig. 1 Experiment 1: Sequence of events of a typical trial and examples of a stimulus display in each condition

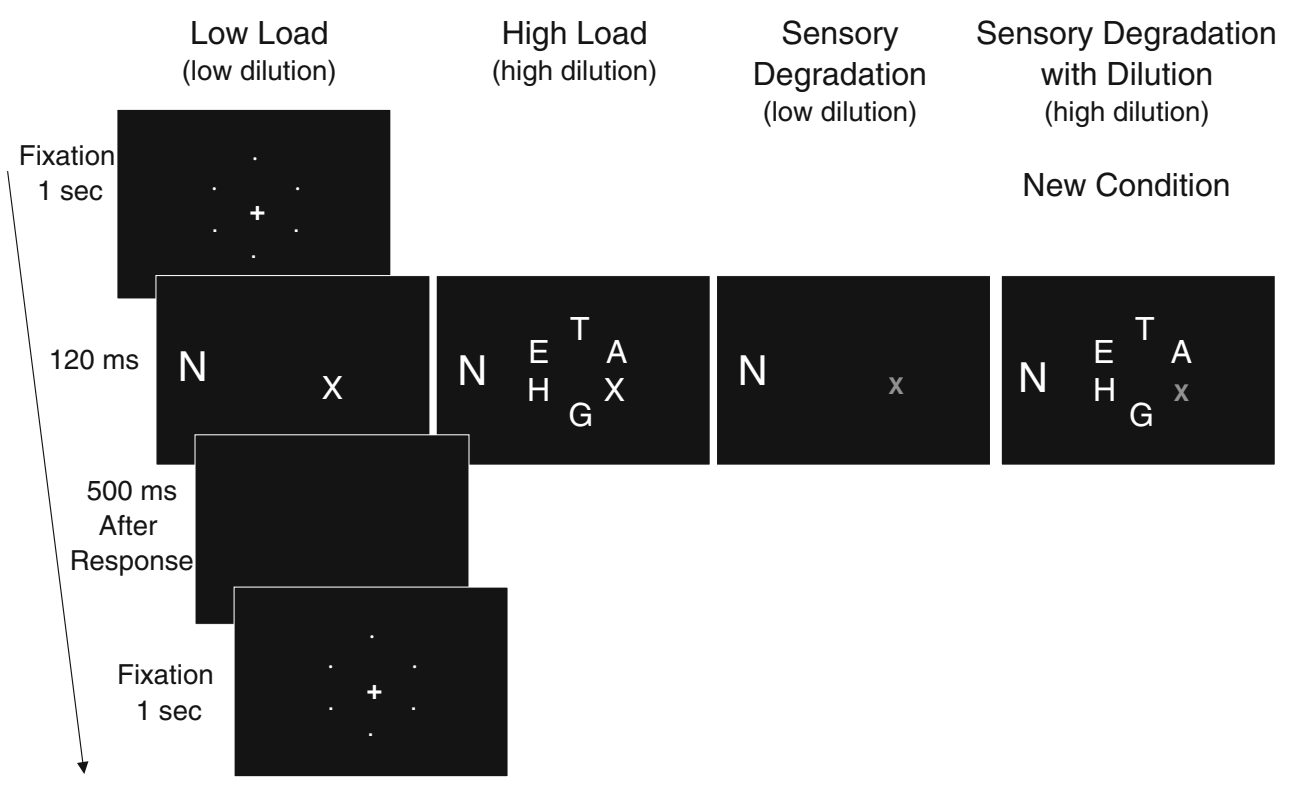

angle in diameter. In the low-load and sensory-degradation conditions, the target letter was presented alone and the other five locations remained empty. In the high-load and sensory-degradation-with-dilution conditions, the other five locations were occupied by five different neutral letters $(\mathrm{G}, \mathrm{E}$, $\mathrm{H}, \mathrm{T}$, and $\mathrm{A}$ ), each subtending $0.72 \mathrm{deg}$ in height and $0.54 \mathrm{deg}$ in width and randomly assigned to the five circular locations. The distractor was centered randomly and equally often $3.2 \mathrm{deg}$ to the left or the right of fixation. The fixation display consisted of a small central cross $(0.38 \times 0.38 \mathrm{deg})$ and six dots marking the six possible target locations. The order of the conditions was randomized across participants, and each condition included 160 trials, preceded by 16 practice trials.

Participants were instructed to respond as quickly and accurately as possible to the target while ignoring the peripheral distractor. They were directed to press the "L" key with their right index finger when the target was an $\mathrm{X}$, and the " $A$ " key with their left index finger when the target was an N. Each trial began with a 1-s fixation display, followed by the stimulus display, which appeared for $120 \mathrm{~ms}$. Error trials were followed by a feedback beep in the practice trials.

\section{Results and discussion}

An overall analysis of variance (ANOVA) carried out on the accuracy data revealed that only the main effect of congruency $[F(1,11)=11.607, p=.006]$ was significant. Neither the main effect for condition $[F(3,33)=0.849, p=.477]$ nor the interaction $[F(3,33)=0.673, p=.579]$ reached statistical significance.

Incorrect responses and responses deviating by more than two standard deviations from the mean in every trial type (e.g., congruent trials in the low-load condition) were removed from the RT analyses (4.2\% of the total trials). An overall Condition $\times$ Congruency within-subjects ANOVA performed on mean RTs revealed that the two main effects and their interaction were all highly significant $[F(3,33)=5.669, p=$ .003 , for condition; $F(1,11)=54.190, p=.000$, for congruency; and $F(3,33)=5.583, p=.003$, for the interaction]. Figure 2 presents the mean RTs for congruent and incongruent displays under the four conditions. Five additional $2 \times 2$ within-subjects ANOVAs were then conducted. The first three replicated the results of Lavie and de Fockert (2003), and the last two tested the predictions of the present experiment. We conducted ANOVAs rather than testing the simple effects of congruency since, as we will show below, it was important to assess congruency effects in the context of corresponding changes in perceptual load (as measured by overall RTs).

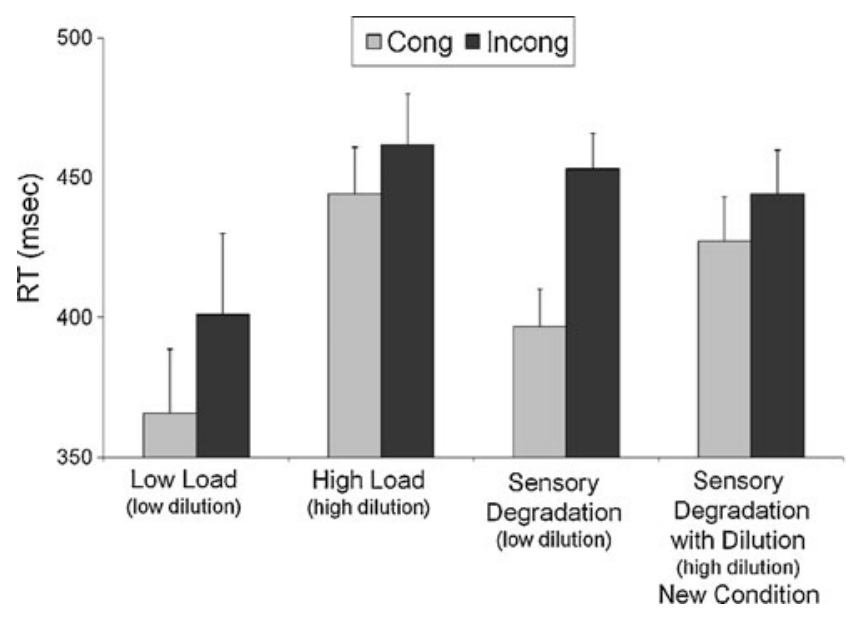

Fig. 2 Experiment 1: Mean RTs for congruent and incongruent displays under the four conditions 
Replications of Lavie and de Fockert (2003)

Perceptual-load effect To assess the traditional perceptualload effect (confounded with dilution) (e.g., Lavie, 1995), we conducted a $2 \times 2$ within-subjects ANOVA comparing congruency effects between the low-load and high-load conditions. The analysis revealed that the target was identified more quickly in the low-load condition than in the high-load condition $[F(1,11)=11.835, p=.006]$ and that congruent displays were responded to more quickly than incongruent displays $[F(1,11)=20.075, p=.001]$. Although, unlike in Lavie and de Fockert (2003), the interaction between these two factors was not significant $[F(1,11)=0.933, p=.355]$, additional planned comparisons did reveal a tendency toward a perceptual-load effect. Analyses of simple effects indicated that while the congruency effect was significant for the low-load condition $[F(1,11)=8.45, p=.014]$, it reached only borderline significance in the high-load condition $[F(1,11)=4.71, p=.053]$.

Sensory-degradation effect The second ANOVA replicated the effect of target degradation on congruency (Lavie \& de Fockert, 2003). The comparison between the congruency effect under the low-load and sensory-degradation conditions indicated that the target was identified more quickly in the low-load condition than in the sensory-degradation condition $[F(1,11)=5.032, p=.006]$ and that congruent displays were responded to more quickly than incongruent ones $[F(1,11)=34.528, p<.001]$. The interaction between these two factors approached significance $[F(1,11)=4.514$, $p=.057]$, indicating that degrading the target resulted in increased distractor processing.

High perceptual load versus target degradation A comparison between the high-load and sensory-degradation conditions revealed that the main effect of congruency was significant $[F(1,11)=50.285, p<.001]$, but the main effect of condition reached only borderline significance $[F(1,11)=4.576$, $p=.056]$. The interaction between the two factors was highly significant $[F(1,11)=15.589, p=.002]$. Hence, as in the results of Lavie and de Fockert (2003), distractor interference was greater in the sensory-degradation condition than in the high-perceptual-load condition.

The results above generally replicated the findings of Lavie and de Fockert (2003) by demonstrating different effects of perceptual load and target degradation on distractor interference. However, it is not clear whether this difference reflects two distinct processes characterizing perceptual versus sensory limitations (Lavie \& de Fockert, 2003) or, instead, the presence of diluting items in the high-perceptual-load condition but not in the sensorydegradation condition. The next two comparisons addressed this issue.

\section{Predictions}

Sensory-degradation effect with and without dilution Comparing the congruency effect between the sensory-degradation condition and the sensory-degradation-with-dilution condition revealed a significant effect of congruency $[F(1,11)=$ $117.933, p=.000]$ but no effect of condition $[F(1,11)=$ $0.495, p=.496]$. Thus, the addition of neutral letters that are clearly distinguishable from the target did not increase perceptual load. Most importantly, as Fig. 2 shows, the substantial congruency effect of $57 \mathrm{~ms}$ obtained in the sensory-degradation condition was markedly reduced, to $17 \mathrm{~ms}$, in the sensory-degradation-with-dilution condition, and this interaction was highly significant $[F(1,11)=$ $38.152, p<.001$ ], demonstrating the potential of neutral letters to dilute distractor interference.

Perceptual load versus sensory degradation when dilution is controlled A comparison between the high-load and sensory-degradation-with-dilution conditions indicated that the effect of congruency was significant $[F(1,11)=10.797$, $p=.007]$, but the effect of condition was not $[F(1,11)=$ $0.742, p=.407]$. Furthermore, the interaction between the two factors was not significant $[F(1,11)=0.027, p=.873]$. This comparison is of major significance to our alternative proposition. The major claim in Lavie and de Fockert's (2003) study was that, while increasing perceptual load largely reduced distractor interference, increasing sensory load substantially increased it. The analysis above shows that when dilution is controlled for by adding neutral diluting items (that are also present in the high-perceptual-load displays) to the sensory-degradation displays, distractor interference is identical. Hence, the opposite effects reported by Lavie and de Fockert were not due to perceptual or sensory limitations per se, but rather to the presence (or absence) of diluting items.

\section{Experiment 2}

The effects of perceptual and sensory loads were assessed by comparing high-perceptual-load and sensory-degradation conditions with the low-perceptual-load condition (Lavie \& de Fockert, 2003). However, in order to ensure precise measures of pure perceptual and sensory limitations, all three conditions must be equated with respect to dilution. This requirement was achieved in the present experiment by presenting the same neutral, potentially diluting items in all three conditions. The present design enabled us (1) to test the prediction that if dilution is properly controlled for, increasing perceptual load will actually enhance distractor processing (similarly to degrading the target) and (2) to compare the pure effects of perceptual load and sensory 
degradation. The original low-load condition was also included in this experiment, as a baseline condition.

Method

\section{Participants}

The participants were 19 undergraduates from Tel Aviv University, who participated to fulfill a course requirement. All had normal or corrected-to-normal vision.

\section{Stimuli and procedure}

The stimuli and procedure were identical to those of Experiment 1, except that the degradation condition was replaced by a low-load-with-dilution condition. The latter condition was identical to the high-load condition, except that the target was marked by $0.42-$ deg horizontal line appearing underneath it. In all conditions, the distractor was slightly enhanced to $1.05 \mathrm{deg}$ in height and $0.75 \mathrm{deg}$ in width, and the displays were presented for $150 \mathrm{~ms}$. In all other respects, the stimuli and procedure were identical to those of Experiment 1. Examples of the stimulus displays are shown in Fig. 3.

\section{Results and discussion}

One participant was excluded from the analyses because his error rate exceeded $30 \%$. An overall ANOVA carried out on the accuracy data revealed that only the main effect of congruency $[F(1,17)=10.956, p=.004]$ was significant. The main effect of condition $[F(3,51)=1.306, p=.283]$ and the interaction between congruency and condition $[F(3$, $51)=1.030, p=.387]$ were not significant.

Incorrect responses and responses deviating by more than two standard deviations from the means in every type of trials $(4.0 \%$ of trials) were removed from the RT analyses. An overall Condition $\times$ Congruency within-subjects ANOVA performed on mean RTs revealed that the two main effects and their interaction were highly significant $[F(3,51)=$ $9.506, p=.000$, for condition; $F(1,17)=23.548, p=.000$, for congruency; and $F(3,51)=6.154, p=.001$, for the interaction]. Figure 4 presents mean RTs for the congruent and incongruent displays under the four conditions. Three additional within-subjects ANOVAs were performed to further investigate the specific effects.

\section{The reversed load effect}

To assess the true, dilution-free, effect of perceptual load, we compared the congruency effect between the highload and the low-load-with-dilution conditions. This comparison showed that the target was identified more quickly in the dilution condition than in the high-load condition $[F(1,17)=6.843, p=.018]$, that congruent displays were responded to more quickly than incongruent ones $[F(1,17)=9.767, p=.006]$, and most importantly, that the interaction between condition and congruency was also significant $[F(1,17)=12.953, p=.002]$, indicating greater distractor interference in the high-load condition than in the
Fig. 3 Experiment 2: Examples of the stimulus displays

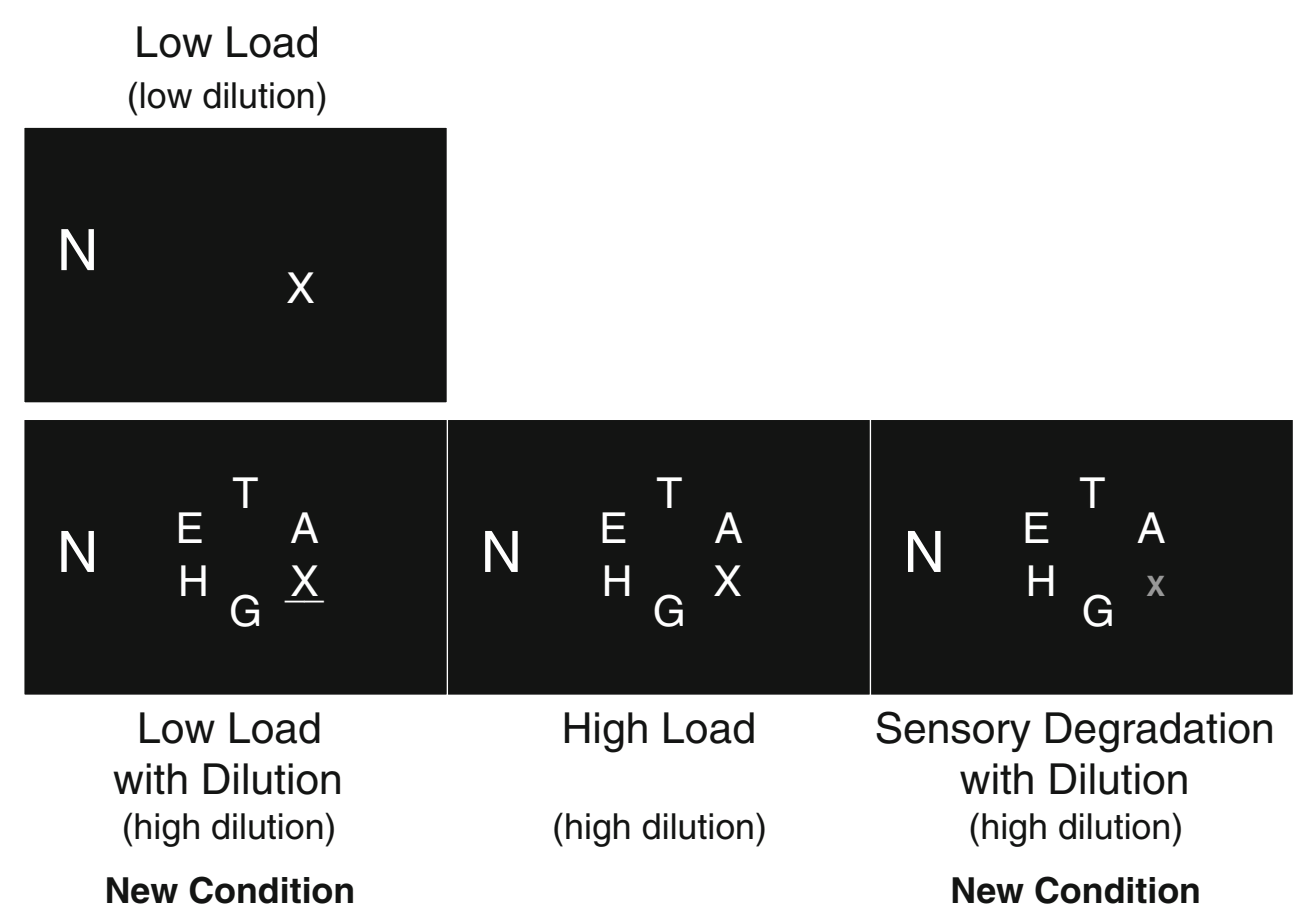




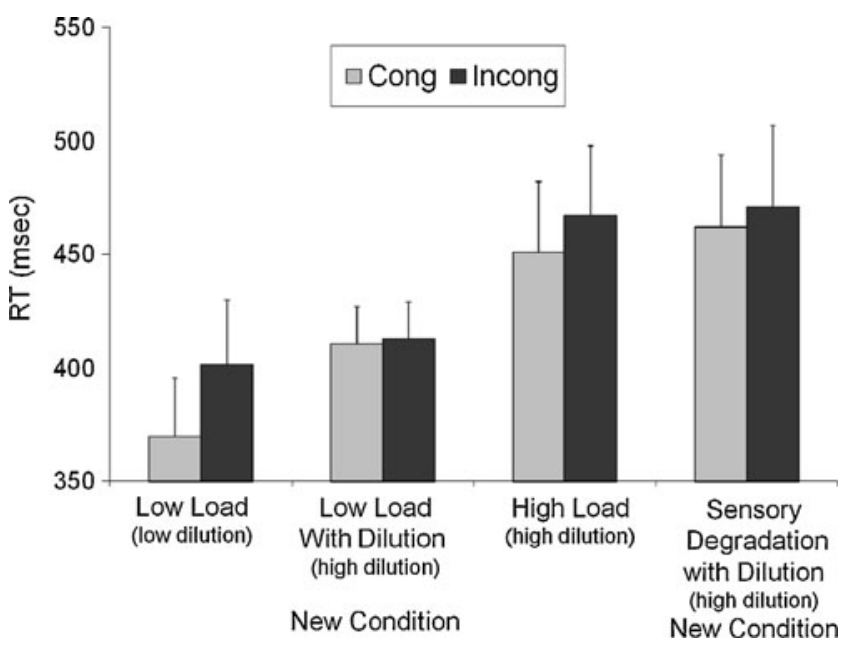

Fig. 4 Experiment 2: Mean RTs for congruent and incongruent displays under the four conditions

low-load-with-dilution condition. This finding shows that when dilution is controlled for-that is, both the low-load and high-load displays contain diluting items - high load produces greater distractor interference than does low load. This result is consistent with the reversed load effect reported by Tsal and Benoni (2010a) and by Wilson et al. (2011).

\section{Perceptual load versus sensory degradation}

To assess the relative effects of perceptual and sensory limitations, we compared the high-load condition with the sensory-degradation-with-dilution condition. The main effect of congruency was significant $[F(1,17)=7.129$, $p=.016]$, but not the effect of condition $[F(1,17)=0.156$, $p=.679]$. The interaction between these two factors was not significant $[F(1,17)=1.249, p=.279]$. Thus, when dilution is controlled for and perceptual and sensory limitations produce similar increases in RTs, they also produce similar increases of distractor processing. This finding supports the conclusion that any increase in task difficulty enhances distractor interference; when dilution is controlled, increasing perceptual load acts exactly like increasing sensory limitations in producing greater distractor processing.

\section{The dilution effect}

A comparison between the low-load and low-loadwith-dilution conditions showed that congruent displays were responded to more quickly than incongruent ones $[F(1,17)=21.786, p<.001]$ and that the target was identified equally quickly in the two conditions $[F(1,17)=$ $2.983, p=.102]$. The latter result indicates that the addition of clearly distinguishable diluting items did not increase perceptual load. Most importantly, the interaction between condition and congruency was highly significant $[F(1,41)=$
23.031, $p<.001]$. Further analyses of simple effects showed that distractor interference in the low-load-with-dilution condition was completely eliminated, as congruent and incongruent displays were responded to equally quickly $[F(1,17)=0.782, p=.388]$.

One could perhaps argue that the low-load and low-loadwith-dilution displays were processed differently, since in the latter but not the former displays attention was required to exclude the diluting items from interfering with target processing, although this processing difference was somehow not reflected in the overall RT. However, it is important to note that, as overall RT is the only available assessment of perceptual load, according to this measure the dilution displays were identical in load to the low-load displays and were substantially different from the highload displays. Hence, the present results are consistent with the previous findings (Benoni \& Tsal, 2010; Tsal \& Benoni, 2010a; Wilson et al., 2011) and lend further support to the conclusion that the traditional effect of perceptual load is due to dilution rather than to perceptual load itself.

\section{General discussion}

The present findings clearly show that when the confounding effect of dilution is controlled, perceptual and sensory manipulations produce the same effects on distractor processing. Our results suggest that the failure of Lavie and de Fockert (2003) to obtain congruency effects in the high-perceptual-load condition (as they obtained in the sensory-degradation condition) was due to the fact that this condition included neutral items that diluted the interference of the distractor. The present Experiment 1 showed that when a degraded target is embedded among clearly distinguishable diluting items, distractor interference is markedly reduced to an extent equal to that in the highperceptual-load condition. Experiment 2 showed that when the high-perceptual-load condition was compared with a lowload condition that also included diluting items, a reversed load effect emerged. That is, perceptual load increased distractor interference to an extent equal to the sensorydegradation condition. Hence, our results suggest that the opposite effects obtained by Lavie and de Fockert could safely be attributed to differences in dilution, since in their study the increase in perceptual load entailed the addition of neutral items capable of diluting distractor processing, whereas their increases in sensory limitations did not.

Over and above the present findings, we argue that there are methodological and theoretical reasons for preferring our proposition that sensory and perceptual limitations are just two aspects of task difficulty, over Lavie and de Fockert's (2003) proposition that the two reflect fundamentally different 
processes. First, predicting the same effects for any increase in task difficulty is more straightforward and parsimonious than predicting diverse effects for sensory (Lavie \& de Fockert, 2003), perceptual (Lavie, 1995), and cognitive (Lavie, Hirst, de Fockert, \& Viding, 2004) manipulations. Second, the effects of task difficulty can directly and precisely be validated by observing consequent changes in RTs. The effects of sensory and perceptual manipulations cannot be easily distinguished, since both produce parallel effects on RTs. Nor can sensory and perceptual operations be distinguished at the antecedent stimulus level, as they comprise a continuum with no clear-cut boundary. In Lavie and de Fockert's various manipulations, they did not rely on explicit criteria for distinguishing sensory and perceptual processing. Third, our proposition relies on more reasonable underlying psychological mechanisms. That is, any increase in task difficulty is likely to impair selective processing, because the greater task demands may capture substantial resources normally needed for inhibiting the interfering distractors. Moreover, more difficult tasks produce longer response latencies, thereby increasing the likelihood of distractor intrusions.

On the other hand, the theoretical bases for the opposite effects produced by sensory and perceptual limitations seem quite problematic. Lavie and de Fockert (2003) argued that sensory manipulations should produce data limits rather than resource limits since, according to Norman and Bobrow (1975), the difficulty produced by degrading the input for a task cannot be compensated for by applying more attentional resources to target processing. However, Lavie and de Fockert's conclusion that sensory manipulations necessarily affect data-limited processes and not resourcelimited processes does not follow from Norman and Bobrow's seminal theory. According to this theory, a degraded stimulus could still entail data limitations as well as resource limitations up to a certain point, when it is so severely degraded that any further allocation of attention will not improve its perception. Evidently, given the relatively high accuracy rates in Lavie and de Fockert's study, it is doubtful that the degraded stimulus had reached such a point at which attention could no longer benefit its perception. Indeed, several studies have shown that attention can improve sensory processing. For example, in a recent study investigating perceptual load, Wegener, Galashan, Markowski, and Kreiter (2006) proposed that attention adjusts neuronal processing to ensure a constant sensory representation of the attended object, as if this object were the only one in the scene. The distinction between datalimited and resource-limited processing could only explain why sensory limitations do not eliminate distractor processing. In order to explain why this condition actually increased distractor processing, Lavie and de Fockert added that increases in sensory limitations provide a larger time window for distractor intrusions. However, exactly the same logic should apply to perceptual load, because nothing is inherently unique to perceptual load that differs from other increases in task difficulty.

Author note This research was supported by Grant 0610714321 from the Israel Science Foundation. Part of this research was presented at the 50th Annual Meeting of the Psychonomic Society, Boston, Massachusetts, in November 2009.

\section{References}

Bavelier, D., Deruelle, C., \& Proksch, J. (2000). Positive and negative compatibility effects. Perception \& Psychophysics, 62, 100-112. doi:10.3758/BF03212064

Beck, D. M., \& Lavie, N. (2005). Look here but ignore what you see: Effects of distractors at fixation. Journal of Experimental Psychology: Human Perception and Performance, 31, 592-607. doi:10.1037/0096-1523.31.3.592

Benoni, H., \& Tsal, Y. (2010). Where have we gone wrong? Perceptual load does not affect selective attention. Vision Research, 50, 1292-1298. doi:10.1016/j.visres.2010.04.018

Carmel, D., Saker, P., Rees, G., \& Lavie, N. (2007). Perceptual load modulates conscious flicker perception. Journal of Vision, 7(14), 14:1-13. doi:10.1167/7.14.14

Dittrich, K., \& Stahl, C. (2011). Nonconcurrently presented auditory tones reduce distraction. Attention, Perception, \& Psychophysics, 73, 714-719. doi:10.3758/s13414-010-0064-8

Forster, S., \& Lavie, N. (2008). Failures to ignore entirely irrelevant distractors: The role of load. Journal of Experimental Psychology: Applied, 14, 73-83. doi:10.1037/1076-898X.14.1.73

Kumada, T., \& Humphreys, G. W. (2002). Early selection induced by perceptual load in a patient with frontal lobe damage: External vs. internal modulation of processing control. Cognitive Neuropsychology, 19, 49-65.

Kyllingsbaek, S., Sy, J. L., \& Giesbrecht, B. (2011). Understanding the allocation of attention when faced with varying perceptual load in partial report: A computational approach. Neuropsychologia, 49, 1487-1497. doi:10.1016/j.neuropsychologia.2010.11.039

Lavie, N. (1995). Perceptual load as a necessary condition for selective attention. Journal of Experimental Psychology: Human Perception and Performance, 21, 451-468. doi:10.1037/0096-1523.21.3.451

Lavie, N. (2006). The role of perceptual load in visual awareness. Brain Research, 1080, 91-100. doi:10.1016/j.brainres.2005.10.023

Lavie, N., \& Cox, S. (1997). On the efficiency of visual selective attention: Efficient visual search leads to inefficient distractor rejection. Psychological Science, 8, 395-398. doi:10.1111/ j.1467-9280.1997.tb00432.x

Lavie, N., \& de Fockert, J. W. (2003). Contrasting effects of sensory limits and capacity limits in visual selective attention. Perception \& Psychophysics, 65, 202-212. doi:10.3758/BF03194795

Lavie, N., \& Fox, E. (2000). The role of perceptual load in negative priming. Journal of Experimental Psychology: Human Perception and Performance, 26, 1038-1052. doi:10.1037/00961523.26.3.1038

Lavie, N., Hirst, A., de Fockert, J. W., \& Viding, E. (2004). Load theory of selective attention and cognitive control. Journal of Experimental Psychology: General, 133, 339-354. doi:10.1037/ 0096-3445.133.3.339

Lavie, N., \& Tsal, Y. (1994). Perceptual load as a major determinant of the locus of selection in visual attention. Perception \& Psychophysics, 56, 183-197. doi:10.3758/BF03213897

Marciano, H., \& Yeshurun, Y. (2011). The effects of perceptual load in central and peripheral regions of the visual field. Visual Cognition, 19, 367-391. doi:10.1080/13506285.2010.537711 
Maylor, E. A., \& Lavie, N. (1998). The influence of perceptual load on age differences in selective attention. Psychology and Aging, 13, 563-573. doi:10.1037/0882-7974.13.4.563

Norman, D. A., \& Bobrow, D. G. (1975). On data-limited and resource-limited processes. Cognitive Psychology, 7, 44-64. doi:10.1016/0010-0285(75)90004-3

Rees, G., Frith, C. D., \& Lavie, N. (1997). Modulating irrelevant motion perception by varying attentional load in an unrelated task. Science, 278, 1616-1619. doi:10.1126/science.278.5343.1616

Rees, G., Frith, C., \& Lavie, N. (2001). Processing of irrelevant visual motion during performance of an auditory attention task. Neuropsychologia, 39, 937-949. doi:10.1016/S00283932(01)00016-1

Thorne, G. L., Dawson, M. E., \& Schell, A. M. (2006). Effects of perceptual load on startle reflex modification at a long lead interval. Psychophysiology, 43, 498-503. doi:10.1111/j.14698986.2006.00420.x
Tsal, Y., \& Benoni, H. (2010a). Diluting the burden of load: Perceptual load effects are simply dilution effects. Journal of Experimental Psychology: Human Perception and Performance, 36, 16451656. doi:10.1037/a0018172

Tsal, Y., \& Benoni, H. (2010b). Much dilution little load in Lavie and Torralbo's (2010) response: A reply. Journal of Experimental Psychology: Human Perception and Performance, 36, 16651668. doi:10.1037/a0021907

Wegener, D., Galashan, F. O., Markowski, D. N., \& Kreiter, A. K. (2006). Selective visual attention ensures constancy of sensory representations: Testing the influence of perceptual load and spatial competition. Vision Research, 46, 3563-3574. doi:10.1016/ j.visres.2006.06.008

Wilson, D. E., Muroi, M., \& MacLeod, C. M. (2011). Dilution, not load, affects distractor processing. Journal of Experimental Psychology: Human Perception and Performance, 37, 319-335. doi:10.1037/a0021433 